\title{
Treatment options of subclinical hyperthyroidism and cardiovascular risk Aglaia Kyrilli ${ }^{1,3}$, Maria Lytrivi ${ }^{1,3}$, Pierre Bel Lassen ${ }^{2}$ and Bernard Corvilain $^{1}$
}

\begin{abstract}
Subclinical hyperthyroidism $(\mathrm{SCH})$ is common and is characterized by laboratory findings of a persistently low TSH level and normal FT4 and FT3 values. The interpretation of studies on the clinical significance of $\mathrm{SCH}$ have been complicated by the fact that the degree and etiology of $\mathrm{SCH}$ varies, but research has suggested that it is associated with osteoporosis, weight changes, adverse cardiovascular effects including an increased risk of atrial fibrillation, and an increased all-cause mortality rate. We discuss $\mathrm{SCH}$ and review the literature on its suspected cardiovascular effects, which are more likely to be seen in the elderly and in patients with more significant degrees of TSH suppression. We then discuss $\mathrm{SCH}$ treatment options in detail and suggest an algorithm for the management of $\mathrm{SCH}$ that takes into consideration the TSH level and the presence of clinical risk factors.
\end{abstract}

\section{Addresses \\ ${ }^{1}$ Division of Endocrinology, Erasme Hospital, Université Libre de Bruxelles, 1070 Brussels, Belgium \\ ${ }^{2}$ Division of Clinical Nutrition, Raymond Poincaré Hospital, 92380 Garches, France}

Corresponding author: Corvilain, Bernard (bcorvila@ulb.ac.be) ${ }^{3}$ A.K and M.L. equally participated to the work.

Current Opinion in Endocrine and Metabolic Research 2018,
2:38-45
This review comes from a themed issue on Thyroid Pathophysiology
Edited by Paolo Beck-Peccoz and Douglas Forrest
For a complete overview see the Issue and the Editorial
Available online 6 February 2018
https://doi.org/10.1016/j.coemr.2018.01.008
2451-9650/O 2018 Elsevier Ltd. All rights reserved.

\section{Keywords}

Subclinical hyperthyroidism, Cardiovascular risk, Thyroid surgery, Radioactive iodine treatment.

\section{Definition}

Subclinical hyperthyroidism $(\mathrm{SCH})$ is a common disease defined exclusively by biological criteria without consideration of the presence or absence of clinical signs and symptoms of hyperthyroidism. It is defined by a persistently low serum TSH $(<0.4 \mathrm{mU} / \mathrm{l})$ associated with FT4 and FT3 values within laboratory reference ranges [1]. Depending on its biological severity, $\mathrm{SCH}$ can be divided into two categories: grade $1 \mathrm{SCH}$, in which the TSH level is only mildly suppressed (typically between 0.1 and $0.39 \mathrm{mU} / \mathrm{L}$ ), and grade $2 \mathrm{SCH}$, in which the TSH is low or undetectable (typically $<0.1 \mathrm{mU} / \mathrm{L}$ ) [2]. The prevalence of SCH varies from 1 to $11 \%$, depending on age, sex, iodine intake and the lower cutoff used to define the normal TSH range. An absolute definition of $\mathrm{SCH}$ is difficult because it seems that each individual has his or her own normal range that is much narrower than the accepted reference range for the general population [4]. Because of the absence of significant symptomatology, most patients with $\mathrm{SCH}$ are detected during routine thyroid screening. The main causes of persistent endogenous $\mathrm{SCH}$ are the presence of autonomous thyroid tissue (toxic adenoma [TA], toxic multinodular goiter [TNG]) and subclinical Graves' disease (GD). The relative prevalence of thyroid autonomy and Graves' disease depends on iodine intake, with thyroid autonomy being by far the predominant cause in areas with mild or moderate iodine deficiency [5-8].

In the absence of major intervention trials, most of the reported effects of SCH are derived from observational studies that must be interpreted with caution given the etiological heterogeneity of SCH; population heterogeneity in terms of age, sex and severity of SCH; the fact that the diagnosis is often based on a single TSH assay; and variability in SCH duration, which is often unknown. This heterogeneity can probably explain the conflicting results across different studies. The main suspected clinical effects of $\mathrm{SCH}$ are changes in bone metabolism, mineral density, body weight and heart disease, including atrial fibrillation [1].

\section{Thyroid hormone effects on the heart}

T4 is secreted by the thyroid gland and converted in the periphery to the biologically active hormone T3. The cellular actions of thyroid hormones are mediated by their binding to nuclear receptors, which act as transcription factors that modulate gene expression. Thyroid hormone receptor affinity is approximately ten-fold higher for T3 than for T4. Thyroid hormones' actions 